\title{
BMJ Open Traditional Chinese acupuncture versus minimal acupuncture for mild-to- moderate knee osteoarthritis: a protocol for a randomised, controlled pilot trial
}

\author{
Ning Sun, ${ }^{1,2}$ Guang-Xia Shi, ${ }^{1}$ Jian-Feng Tu, ${ }^{1}$ Yong-Ting Li, ${ }^{1}$ Li-Wen Zhang, ${ }^{2}$ \\ Yan Cao, ${ }^{2}$ Yi Du, ${ }^{3}$ Jing-Jie Zhao, ${ }^{3}$ Da-Chang Xiong, ${ }^{4}$ Hai-Kun Hou, ${ }^{4}$ Cun-Zhi Liu ${ }^{1}$
}

To cite: Sun N, Shi G-X, Tu J-F, et al. Traditional Chinese acupuncture versus minimal acupuncture for mild-to-moderate knee osteoarthritis: a protocol for a randomised, controlled pilot trial. BMJ Open 2016:6: e013830. doi:10.1136/ bmjopen-2016-013830

- Prepublication history for this paper is available online. To view these files please visit the journal online (http://dx.doi.org/10.1136/ bmjopen-2016-013830).

Received 10 August 2016 Accepted 7 October 2016

CrossMark

For numbered affiliations see end of article.

Correspondence to Dr Cun-Zhi Liu; Icz623780@126.com

\section{ABSTRACT}

Introduction: Knee osteoarthritis (KOA) is one of the most common musculoskeletal disorders. Acupuncture is a popular form of complementary medicine for musculoskeletal conditions, although the evidence is inconclusive. Our objective is to evaluate the efficacy of traditional Chinese acupuncture for pain relief and function improvement in mild-to-moderate knee osteoarthritis (TCAKOA) participants.

Methods/analysis: 42 patients will be recruited who have been diagnosed with mild-to-moderate KOA and randomly allocated in equal proportions to traditional Chinese acupuncture or minimal acupuncture. They will receive acupuncture for 24 sessions over 8 weeks. The primary end point is success rate, which will be calculated according to a change from baseline in Western Ontario and McMaster Universities Osteoarthritis Index pain and function scores at 8 weeks. Secondary end points include pain and function measurement, global change, the quality of life and the use of non-steroidal anti-inflammatory drugs (Celebrex, Pfizer) at 8, 16 and 26 weeks.

Ethics and dissemination: Ethical approval of this study has been granted by the Research Ethical Committee of Beijing Hospital of Traditional Chinese Medicine Affiliated to Capital Medical University (permission number: 2016BL-010-02). Written informed consent will be obtained from all participants. Outcomes of the trial will be disseminated through peer-reviewed publications.

Trial registration number: ISRCTN14016893; Preresults.

\section{BACKGROUND}

Knee osteoarthritis (KOA) is one of the most common musculoskeletal disorders, ${ }^{1}$ which features as a protracted course of disease. A systematic review shows that the prevalence of $\mathrm{KOA}$ is $27.3 \%$ in women and $21.0 \%$ in men. ${ }^{2}$ A cross-sectional study with 9512 participants aged 50 years or older shows that the prevalence of radiographic KOA was $43.8 \%$ in women and $21.1 \%$ in men in South Korea. ${ }^{3} \mathrm{KOA}$ is one of the leading causes of pain and global disability.

The objective of treating KOA is the alleviation of pain and improving quality of life. Five guidelines ${ }^{1}{ }^{4-6}$ have evaluated treatment effects on key outcomes of KOA (including pain, function and disability). Pharmacologic agents, comprising non-opioid/opioid oral, non-steroidal anti-inflammatory drugs (NSAIDs) oral, intra-articular steroid, topical analgesics and hyaluronate injections are normally used but may be associated with significant adverse reactions (such as peptic ulcer, hypertension and renal damage). ${ }^{6-8}$ Guidelines emphasise the potential role of non-pharmacologic treatment, such as aerobic exercise, electrical nerve stimulation, acupuncture in the treatment. Effective alternatives to pharmacological are therefore desirable.

Traditional Chinese acupuncture (TCA) is a popular form of complementary medicine. In 2005, Germany, Witt and colleagues showed that 8 weeks of the semistandardised acupuncture treatment had significantly alleviated the patient's pain and dysfunction contrasted to the minimal acupuncture (MA) treatment and no treatment condition. ${ }^{9}$ A meta-analysis showed that acupuncture could be considered to be an effective physical treatment for KOA. ${ }^{10}$ However, in the October 2014 publication of JAMA, Dr Hinman et al conducted a Zelen design clinical trial to investigate acupuncture for patients suffering from chronic knee pain. The investigation declared that acupuncture did not convey more advantages compared to sham or better function in sufferers with mild or harsh chronic knee pain. ${ }^{11}$ However, flaws may exist in the trial design, statistics, interpretation of the results. ${ }^{12-26}$ First of all, participants aged $\geq 50$ years with 
moderate-to-severe chronic knee pain have been recruited. These inclusion criteria may be more suitable for arthroscopic or joint replacement therapy according to the guidance. ${ }^{14}$ Second, acupuncture intervention is $8-12$ sessions in total. The dosage for acupuncture is far from sufficient. ${ }^{14} 16$ Third, the team registered trial as studying laser acupuncture, instead of traditional acupuncture. $^{27}$ Researchers had changed the main aim selectively. ${ }^{14}{ }^{26}$ At present, there is a controversy over whether the acupuncture has benefit for KOA. $^{1}{ }^{4}$ Therefore, our aim is to investigate the intensive TCA for participants with mild-to-moderate KOA.

\section{MATERIALS AND METHODS}

\section{Study design}

The study proposes a two-arm, randomised, clinical pilot trial. We will enrol patients from Beijing Hospital of Traditional Chinese Medicine Affiliated to Capital Medical University, Beijing Friendship Hospital and Beijing Jishuitan Hospital. The trial has been registered with ISRCTN at Current Controlled Trials (ISRCTN14016893). Some recruitment strategies include radio and print advertisements through the local web sites and community centre as well as recruiters with general practitioners. The intervention includes 24 sessions of acupuncture and 3 times follow-up (figure 1 and table 1 ).

\section{Inclusion criteria}

1. Age $45-75$ years (either sex)

2. Chronic knee pain for the last 6 months

3. Morning stiffness $\leq 30 \mathrm{~min}$.

The above-mentioned criteria are consistent with the National Institute for Health and Clinical Excellence (NICE) Guidelines 2014 Edition. ${ }^{4}$

4. Radiologic confirmation of KOA (Kellgren-Lawrence grade II or III ${ }^{28}$ ).

\section{Exclusion criteria}

1. Recent acupuncture

2. Other sickness impact the knee

3. On surgical operation list

4. Neurologic as well as psychiatric diseases

5. Severe coagulopathy

6. Breastfeeding or pregnancy

7. Not fitting to take the NSAID (Celebrex, Pfizer) provided.

Figure 1 Trial flow chart. KOA, knee osteoarthritis. 
Table 1 Time to visit and data collection

\begin{tabular}{|c|c|c|c|c|c|}
\hline & \multirow{2}{*}{$\begin{array}{l}\text { Baseline } \\
-1 \text { day }\end{array}$} & \multicolumn{2}{|c|}{ Treatment phase } & \multicolumn{2}{|c|}{ Follow-up phase } \\
\hline & & 0 day & 8 weeks & 16 weeks & 26 weeks \\
\hline \multicolumn{6}{|l|}{ Patients } \\
\hline Informed consent & $\times$ & & & & \\
\hline Sign informed consent & & $x$ & & & \\
\hline Medical history & $x$ & & & & \\
\hline Physical examination & $\times$ & & & & \\
\hline Randomisation & & $x$ & & & \\
\hline Intervention & \multicolumn{5}{|c|}{24 sessions of TCA } \\
\hline TCA group $(n=21)$ & & & & & \\
\hline Comparisons & \multicolumn{5}{|c|}{24 sessions of MA } \\
\hline MA group $(n=21)$ & & & & & \\
\hline \multicolumn{6}{|l|}{ Outcomes } \\
\hline WOMAC & & $x$ & $\times$ & $x$ & $x$ \\
\hline KOOS & & $x$ & $x$ & $x$ & $x$ \\
\hline VAS & & $\times$ & $\times$ & $\times$ & $\times$ \\
\hline SF-12 & & $x$ & $x$ & $x$ & $x$ \\
\hline The use of NSAIDs & & & $x$ & $x$ & $x$ \\
\hline \multicolumn{6}{|l|}{ Participant safety } \\
\hline Adverse events & & $x$ & $\times$ & $x$ & $\times$ \\
\hline
\end{tabular}

For bilaterally eligible knees, the most symptomatic side will be evaluated in the course of the study.

\section{Randomisation and allocation concealment}

Eligible patients will be randomly assigned to the TCA group or the MA group in a ratio of 1:1 through central automated allocation procedures. An independent statistician generates randomisation sequence by using the SAS V.9.1.3 statistical package (SAS Institute, Cary, North Carolina, USA). Acupuncturists will not involve in the process of randomisation.

The research assistants who collect data, the statisticians who assess outcomes and make statistical analysis will be blinded to group assignment. Participants will not be disclosed information regarding the allocation. Administrators will not be blinded because of the nature of intervention.

\section{Interventions}

The acupuncture protocol follows the CONSORT ${ }^{29}$ and STRICTA. ${ }^{30}$ All acupuncturists have Chinese medicine practitioner licenses, and they have been qualified for at least 10 years. All acupuncturists will receive training in the application of MA. Celebrex will give to participants if their pain intensity $\geq 80$ on a $10 \mathrm{~cm}$ visual analogue scale (VAS). ${ }^{31}$

The protocol specifies the intervention of acupuncture to be a $20 \mathrm{~min}$ treatment which is applied 3 times weekly for 8 weeks, with 24 sessions in total permitted. Disposable, sterile steel, $0.30 \times 25 \mathrm{~mm}$ or $0.30 \times 40 \mathrm{~mm}$ needles (Huatuo disposable acupuncture needle, Suzhou Medical Co., Jiangsu, China) will be used in two groups.

\section{TCA group}

Acupuncture points are selected on traditional Chinese Medicine theory of the ' $\mathrm{Bi}$ ' syndrome. These points are composed of 10 local points (ST34, ST35, ST 36, EX-LE2, EX-LE5, GB33, GB34, SP9, SP10, LV8) and 11 distal points (GB31, GB36, GB39, GB41, ST40, ST41, LR3, BL60, SP6, KI3, LI4) (figure 2). Physicians can choose five to six local points and three to four distal points. Needles will be making an optimum insertion into the skin. Acupuncturists are instructed to achieve 'De Qi', and needles will be stimulated manually at least $10 \mathrm{~s}$.

\section{MA group}

Non-acupoints in a superficial puncture $(2 \mathrm{~mm}$ in depth) will be performed in the MA group. Treatment is standardised needling without manual stimulation at seven points at certain distances from TCA group points (table 2). The MA procedure will be given on the same schedule as the TCA group.

\section{OUTCOMES}

\section{Primary outcome measurement}

Success rate will be calculated according to a change from baseline in Western Ontario and McMaster Universities Osteoarthritis Index (WOMAC) ${ }^{32} 33$ pain and function scores at 8 weeks. WOMAC function subscale (17 items, scored from 0 to 68 ) and pain subscale (5 items, scored from 0 to 20) with higher scores represent worse pain and function.

\section{Secondary outcome measurement}

Knee pain will be assessed by WOMAC pain subscale and Visual Analogue Scale (VAS, 0-100, higher scores 

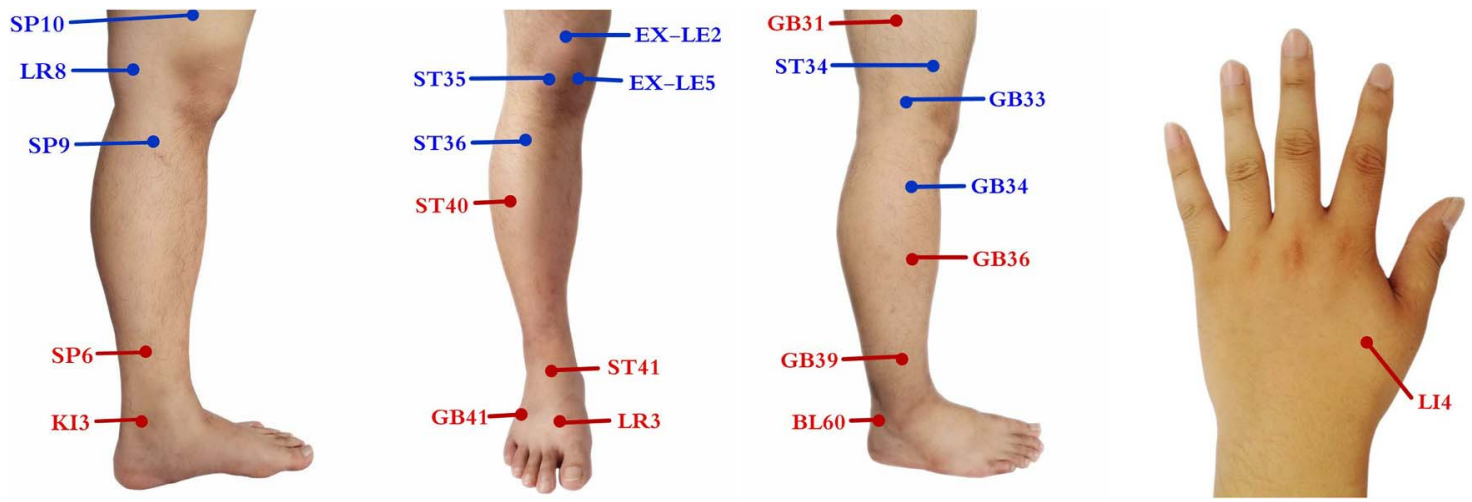

Figure 2 The points used in the TCA group. TCA, traditional Chinese acupuncture.

\begin{tabular}{|c|c|}
\hline $\begin{array}{l}\text { Sham } \\
\text { acupuncture } \\
\text { points }\end{array}$ & Location \\
\hline MP1 & $\begin{array}{l}\text { Ulnar margin of forearm, midpoint o } \\
\text { the connecting line between the } \\
\text { rasceta head and condylus medialis } \\
\text { humeri }\end{array}$ \\
\hline MP2 & $\begin{array}{l}2 \text { cun above the malleolus lateralis, } \\
\text { between the gall bladder meridian } \\
\text { and stomach meridian on the distal } \\
\text { part of the fibula }\end{array}$ \\
\hline MP3 & $\begin{array}{l}2 \text { cun above the malleolus medialis, } \\
\text { in the centre of the tibia surface } \\
\text { area (intracutaneous without periost } \\
\text { contact, in the direction towards the } \\
\text { knee) }\end{array}$ \\
\hline MP4 & $\begin{array}{l}\text { Midpoint of the connecting line } \\
\text { between ST36 and GB34 }\end{array}$ \\
\hline MP5 & $\begin{array}{l}6 \text { cun above the upper edge of the } \\
\text { patella (between the spleen and } \\
\text { stomach meridian) }\end{array}$ \\
\hline MP6 & $\begin{array}{l}5 \text { cun above the upper edge of the } \\
\text { patella (between the spleen and } \\
\text { stomach meridian) }\end{array}$ \\
\hline MP7 & $\begin{array}{l}4 \text { cun above the upper edge of the } \\
\text { patella (between the spleen and } \\
\text { stomach meridian) }\end{array}$ \\
\hline MP8 & $\begin{array}{l}1 \text { cun under the tibia head, in the } \\
\text { medial edge of leg }\end{array}$ \\
\hline MP9 & $\begin{array}{l}\text { Midpoint of the connecting line } \\
\text { between GB40 and ST4 } 1\end{array}$ \\
\hline MP10 & $\begin{array}{l}3 \text { cun above the medial edge of } \\
\text { calcaneal }\end{array}$ \\
\hline
\end{tabular}

One 'cun' is defined according to the rules of traditional Chinese medicine as the width of the interphalangeal joint of patient's thumb.

MA, minimal acupuncture.

representing worse pain). WOMAC function subscale will be used to measure physical function. Knee injury and Osteoarthritis Outcome Score (KOOS 0-100, higher scores indicating better function) subscales comprise pain, symptoms, activities of daily living and quality of life. ${ }^{34}$ Health-related quality of life will use the 12-item Short Form Health Survey (SF-12 0-100, higher scores representing better quality of life). ${ }^{35}$ The use of the NSAIDs (Celebrex, Pfizer) will be at 8, 16 and 26 weeks.

Adverse events will be monitored and reported by acupuncturists via open-ended questioning. Patients will be suggested to state any adverse circumstances they go through, comprising discomfort or bruise in the locations pierced by needle, nausea or the feeling of faint after the acupuncture treatment. Every crucial sign and adverse events are going to be investigated and recorded during every visit.

\section{Sample size}

The purpose is to accumulate clinical data, obtain the outcome data of the intervention method and prove the feasibility of the study protocol. Forty-two patients will be selected as the sample size according to clinical experience.

\section{Statistical analysis}

The results will be analysed by using the SPSS software (SPSS V.12.0 KO for Windows). The accepted level of significance will be $\mathrm{p}<0.05$. Measurement data were expressed by mean number $\pm \mathrm{SD}$, enumeration data expressed as a percentage.

The statistical analysis will be carried out based on the theory of intention-to-treat (ITT) analysis as well as perprotocol (PP) analysis. In the case of ITT analysis, missing data will be replaced according to the principle of the last observation carried forward and the maximum likelihood regression analysis. PP analysis will be conducted with patients who have received treatments $>16$ times and complete the case report form (CSF). $\chi^{2}$ Test is going to be performed for the situation of proportions; meanwhile, the analysis of independent sample t-tests is going to be conducted to examine the baseline discrepancies between the two groups. The significance of the differences in the various data in each group will be analysed with a paired t-test. On the basis of the baseline and temporary analgesic medicine dosage adjustment, continuous measurement results will 
be analysed using covariance test, and logistic regression analysis will be used for the two classification outcomes. Above two analyses will be present as difference in means or advantage ratio with $95 \%$ CIs.

\section{Ethics and dissemination}

The protocol has been registered to ClinicalTrials.gov registry. Any revisions about the protocol will be documented in the ClinicalTrials.gov registry. Written informed consent will be obtained from all participants. The patients will be given adequate time to raise questions and to consider whether or not to involve in the study. We are going to publish the results of this trial in a peer-reviewed clinical journal to have widespread dissemination.

\section{DISCUSSION}

KOA is a common public health problem and a leading cause of disability. The results of this pilot study are going to concentrate on patients suffering from mild-to-moderate KOA and will investigate whether acupuncture can be a practicable and efficient therapy.

A suitable control group is critical for a well-designed clinical trial. On the basis of the literature review as well as clinical experiences, the acupoints in the MA group do not therapeutically affect KOA. Additionally, the dosage for acupuncture is sufficient. The protocol specifies the intervention of acupuncture to be a $20 \mathrm{~min}$ treatment which is applied 3 times weekly for 8 weeks, with 24 sessions in total permitted. Moreover, according to generality for the trial, the wide inclusion criteria will render it more possible that the participants fairly stand for those who have mild-to-moderate KOA. One potential limitation of this study is that acupuncturists are not blinded because of the nature of intervention. However, acupuncturists will not relate to the outcome assessments or data analyses.

The pilot trial will supply the clinical foundation as well as data that are demanded for evaluating the practicability for a large-scale RCT trial in the future.

\section{Trial status}

This trial is currently recruiting participants.

\section{Author affiliations}

${ }^{1}$ Department of Acupuncture and Moxibustion, Beijing Hospital of Traditional Chinese Medicine affiliated to Capital Medical University, Beijing, China ${ }^{2}$ Department of Medicine, School of Medicine, Shandong University of Traditional Chinese Medicine, Jinan, China

${ }^{3}$ Department of Traditional Chinese Medicine, Beijing Friendship Hospital, Capital Medical University, Beijing, China

${ }^{4}$ Department of Acupuncture and Moxibustion, Beijing Jishuitan Hospital, Peking University, Beijing, China

Contributors NS, C-ZL and G-XS conceived of the study. C-ZL, G-XS, NS, J-FT and Y-TL initiated the study design. YD, J-JZ, D-CX and H-KH helped with its implementation. NS, G-XS, J-FT, L-WZ and YC drafted and critically revised the manuscript for important intellectual content. C-ZL sought funding and ethical approval. All authors contributed to the refinement of the study protocol and approved the final manuscript.
Funding This work was supported by Beijing Municipal Administration of Hospitals Clinical Medicine Development of Special Funding Support (code: XMLX201607)

Competing interests None declared.

Patient consent Obtained.

Ethics approval Beijing Hospital of Traditional Chinese Medicine Affiliated to Capital Medical University.

Provenance and peer review Not commissioned; peer reviewed for ethics and funding approval prior to submission.

Open Access This is an Open Access article distributed in accordance with the terms of the Creative Commons Attribution (CC BY 4.0) license, which permits others to distribute, remix, adapt and build upon this work, for commercial use, provided the original work is properly cited. See: http:// creativecommons.org/licenses/by/4.0/

\section{REFERENCES}

1. Hochberg MC, Altman RD, April KT, et al. American College of Rheumatology 2012 recommendations for the use of nonpharmacologic and pharmacologic therapies in osteoarthritis of the hand, hip, and knee. Arthritis Care Res (Hoboken) 2012;64:465-74.

2. Pereira $\mathrm{D}$, Peleteiro B, Araújo J, et al. The effect of osteoarthritis definition on prevalence and incidence estimates: a systematic review. Osteoarthr Cartil 2011;19:1270-85.

3. Lee S, Kim SJ. Prevalence of knee osteoarthritis, risk factors, and quality of life: the Fifth Korean National Health and Nutrition Examination Survey. Int $J$ Rheum Dis Published Online First: 18 Nov 2015. DOI: 10.1111/1756-185X.12795

4. NICE. Osteoarthritis: care and management. Guidance and guidelines. https://www.nice.org.uk/guidance/cg177 (accessed 31 Jul 2016).

5. Jordan KM, Arden NK, Doherty M, et al. EULAR recommendations 2003: an evidence based approach to the management of knee osteoarthritis: report of a Task Force of the Standing Committee for International Clinical Studies Including Therapeutic Trials (ESCISIT). Ann Rheum Dis 2003:62:1145-55.

6. Zhang W, Nuki G, Moskowitz RW, et al. OARSI recommendations for the management of hip and knee osteoarthritis: part III: changes in evidence following systematic cumulative update of research published through January 2009. Osteoarthr Cartil 2010;18:476-99.

7. Richette P. How safe is acetaminophen in rheumatology? Joint Bone Spine 2014;81:4-5.

8. Henry D, McGettigan P. Epidemiology overview of gastrointestinal and renal toxicity of NSAIDs. Int J Clin Pract Suppl 2003:43-9.

9. Witt $C$, Brinkhaus $B$, Jena $S$, et al. Acupuncture in patients with osteoarthritis of the knee: a randomised trial. Lancet 2005;366:136-43.

10. Corbett MS, Rice SJC, Madurasinghe V, et al. Acupuncture and other physical treatments for the relief of pain due to osteoarthritis of the knee: network meta-analysis. Osteoarthr Cartil 2013;21:1290-8.

11. Hinman RS, McCrory P, Pirotta M, et al. Acupuncture for chronic knee pain: a randomized clinical trial. JAMA 2014;312:1313-22.

12. Hinman RS, Pirotta M, Bennell KL. Treating chronic knee pain with acupuncture-reply. JAMA 2015;313:628-9.

13. Li YM. Treating chronic knee pain with acupuncture. JAMA 2015;313:628.

14. Lao L, Yeung WF. Treating chronic knee pain with acupuncture. JAMA 2015;313:627-8.

15. Fleckenstein J, Banzer W. Treating chronic knee pain with acupuncture. JAMA 2015;313:627.

16. He H. Treating chronic knee pain with acupuncture. JAMA 2015;313:626.

17. Baxter GD, Tumilty S. Treating chronic knee pain with acupuncture. JAMA 2015;313:626-7.

18. McGlothlin $A E$, Lewis RJ. Minimal clinically important difference: defining what really matters to patients. JAMA 2014;312:1342-3.

19. Kmietowicz $Z$. Acupuncture does not improve chronic knee pain, study finds. BMJ 2014;349:g5899.

20. Yang MX, Yang J, Zheng $\mathrm{H}$, et al. Comments on "Acupuncture for chronic knee pain: a randomized clinical trial" from Journal of the American Medical Association. Zhongguo Zhen Jiu 2015;35:299-304.

21. White $A$, Cummings M. Acupuncture for knee osteoarthritis: study by Hinman et al represents missed opportunities. Acupunct Med 2015;33:84-6. 
22. Zhang Q, Yue J, Lu Y. Acupuncture treatment for chronic knee pain: study by Hinman et al underestimates acupuncture efficacy. Acupunct Med 2015;33:170.

23. Hinman RS, Forbes A, Williamson E, et al. Acupuncture for chronic knee pain: a randomised clinical trial. Authors' reply. Acupunct Med 2015;33:86-8.

24. Fan AY. The methodology flaws in Hinman's acupuncture clinical trial, Part III: sample size calculation. J Integr Med 2015;13:209-11.

25. Fan AY. The methodology flaws in Hinman's acupuncture clinica trial, Part II: Zelen design and effectiveness dilutions. J Integr Med 2015;13:136-9.

26. Fan AY. The methodology flaws in Hinman's acupuncture clinical trial, part I: design and results interpretation. J Integr Med 2015;13:65-8.

27. Hinman RS, McCrory P, Pirotta M, et al. Efficacy of acupuncture for chronic knee pain: protocol for a randomised controlled trial using a Zelen design. BMC Complement Altern Med 2012;12:161.

28. Kellgren JH, Lawrence JS. Radiological assessment of osteo-arthrosis. Ann Rheum Dis 1957;16:494-502.31.

29. Schulz KF, Altman DG, Moher D, et al. CONSORT 2010 statement: updated guidelines for reporting parallel group randomised trials. BMJ 2010;340:c332.
30. MacPherson H, Altman DG, Hammerschlag R, et al. Revised STandards for Reporting Interventions in Clinical Trials of Acupuncture (STRICTA): extending the CONSORT statement. PLOS Med 2010;7:e1000261.

31. Liu CZ, Xie JP, Wang LP, et al. A randomized controlled trial of single point acupuncture in primary dysmenorrhea. Pain Med 2014:15:910-20.

32. Bellamy N, Buchanan WW, Goldsmith $\mathrm{CH}$, et al. Validation study of WOMAC: a health status instrument for measuring clinically important patient relevant outcomes to antirheumatic drug therapy in patients with osteoarthritis of the hip or knee. J Rheumatol 1988;15:1833-40.

33. Goldsmith $\mathrm{CH}$, Boers $\mathrm{M}$, Bombardier $\mathrm{C}$, et al. Criteria for clinically important changes in outcomes: development, scoring and evaluation of rheumatoid arthritis patient and trial profiles. OMERACT Committee. J Rheumatol 1993;20:561-5.

34. Roos EM, Roos HP, Lohmander LS, et al. Knee injury and Osteoarthritis Outcome Score (KOOS)_development of a self-administered outcome measure. J Orthop Sports Phys Ther 1998;28:88-96.

35. Ware J, Kosinski M, Keller SD. A 12-item Short-Form Health Survey: construction of scales and preliminary tests of reliability and validity. Med Care 1996;34:220-33. 